\title{
Contralateral Acute Subdural Hematoma Following Traumatic Acute Subdural Hematoma Evacuation
}

\author{
-Case Report-
}

\author{
Jian SHEN, ${ }^{1}$ Zuoxu FAN, ${ }^{1}$ Tao JI, ${ }^{1}$ Jianwei PAN, ${ }^{1}$ \\ Yongqing ZHOU, ${ }^{1}$ and Renya $\mathrm{ZHAN}^{1}$ \\ ${ }^{1}$ Department of Neurosurgery, First Affiliated Hospital, College of Medicine, \\ Zhejiang University, Hangzhou, Zhejiang Province, PRC
}

\begin{abstract}
Contralateral acute subdural hematoma (ASDH) occurring after removal of traumatic ASDH is a rare, but nearly devastating postoperative complication. We treated a 26-year-old male who developed a contralateral ASDH shortly after craniectomy for evacuation of a traumatic ASDH. Burr-hole craniotomy was performed before decompressive craniectomy, and the bleeding source was a cortex artery within the frontal lobe contusion. Despite supportive therapy with barbiturate and mild hypothermia he expired 3 days later of brain death. Literature review suggests that the old are more susceptible to contralateral ASDH following evacuation of traumatic ASDH. Contralateral ASDH following evacuation of traumatic ASDH is a rare but potentially lethal complication, so neurosurgeons should try to detect such contralateral hematoma formation and prevent clinical deterioration.
\end{abstract}

Key words: acute subdural hematoma, decompressive craniectomy, postoperative hemorrhage

\section{Introduction}

Patients with acute subdural hematoma (ASDH) suffer high mortality rates, despite modern surgical, medical, and technological advances. ${ }^{14,15,19)}$ Decompressive craniectomy in the management of patients with ASDH have been justified by various reports, ${ }^{3,8,20)}$ but complications secondary to surgical decompression are likely to adversely affect outcome..$^{9,16,20)}$ Contralateral ASDH occurring after removal of traumatic ASDH have been reported recently as a rare but potentially lethal postoperative complication which requires consecutive operations. Delayed contralateral ASDH may manifest as intraoperative brain swelling, postoperative neurological deterioration, or intractably elevated intracranial pressure (ICP). If unrecognized, this disorder can cause devastating consequences. We report a case of contralateral ASDH occurring after hematoma evacuation in a patient with traumatic ASDH, and discuss this rare phenomenon.

\section{Case Report}

A 26-year-old male suffered severe left temporo-occipital brain injury in a car accident, and was admitted to our hospital with progressive headache and irritability. On admission, clinical examination demonstrated poor orientation in time and place, pupils of equal size with normal light response, Glasgow Coma Scale (GCS) score of 14, and

Received January 10, 2012; Accepted April 11, 2012 a left temporo-occipital scalp hematoma. Specific hematological investigations, liver function tests, and coagulation profile were all within the normal ranges. In particular, the platelet count was demonstrated as $316 / \mathrm{mm}^{3}$ (normal $100-300 / \mathrm{mm}^{3}$ ), whereas coagulation studies revealed prothrombin time (PT) of 10.4 sec (normal 10.0-13.5 sec), activated partial thromboplastin time of $29.5 \mathrm{sec}$ (normal 22.0-36.0 sec), and thrombin time of $17.2 \mathrm{sec}$ (normal 14.5-21.5 sec). Tests found D-dimer of $405 \mu \mathrm{g} / \mathrm{l}$ (normal 0-500 $\mu \mathrm{g} / \mathrm{l}$ ), fibrinogen of $3.4 \mathrm{~g} / \mathrm{l}$ (normal 2.0-4.0 g/l), and PT-international normalized ratio (INR) of 0.95 (normal $0.80-1.15$ ). Furthermore, the patient was not receiving any antiplatelet or anticoagulant agents, and had no family history of bleeding diathesis. His medical history was free of severe systematic disease. Emergent brain computed tomography (CT) revealed a right frontal small contusion and subarachnoid hemorrhage without midline shift (Fig. 1A, D). Mannitol infusion to control the increased ICP was not directly recommended, and observations were performed carefully. However, his level of consciousness decreased rapidly to GCS of 8 within half an hour, and his left and right pupils were $3 \mathrm{~mm}$ and $5 \mathrm{~mm}$ in diameter without light response. Primary resuscitation and stabilization therapy were performed. Repeat brain CT demonstrated a right fronto-temporal ASDH (4 $\mathrm{mm}$ maximum width) and a left frontal small contusion associated with severe brain swelling and midline shift of $6 \mathrm{~mm}$ (Fig. 1B, E).

Burr-hole craniotomy and dural incision in the right temporal region followed by decompressive craniectomy 
and hematoma evacuation were performed within 3 hours of the accident. The brain showed considerable swelling and dural grafting was used. Postoperative CT revealed contralateral fronto-temporo-parietal ASDH (10 mm maximum width) with a midline shift of $15 \mathrm{~mm}$ (Fig. 1C, F). The patient was rapidly returned to the operating room within 5 minutes. Burr-hole craniotomy was also performed rapidly before decompressive craniectomy, and the bleeding source was a cortex artery within the frontal lobe contusion. Fresh frozen plasma was administered in view of resuscitation and blood volume loss. The intraoperative check coagulation profile did not demonstrate trauma-in-
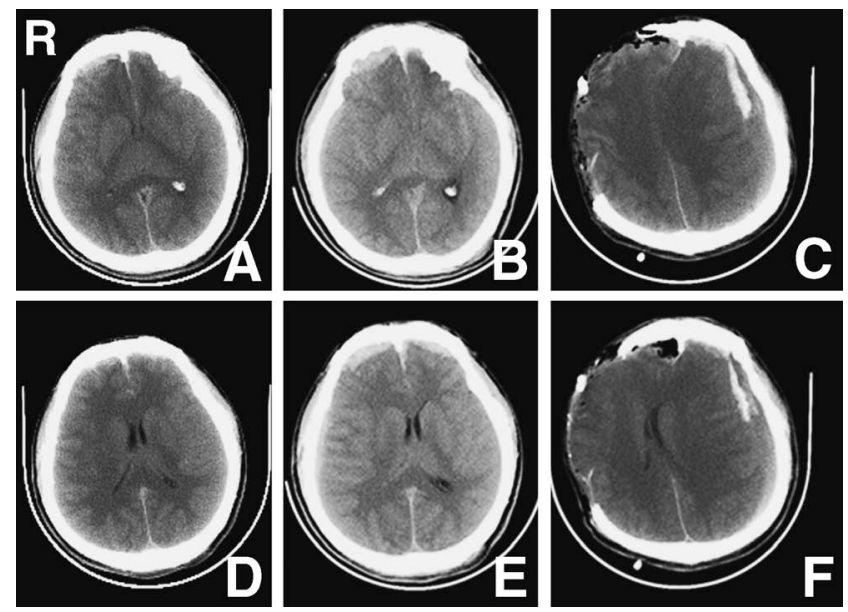

Fig. 1 A, D: Axial computed tomography (CT) scans on admission showing a right frontal small contusion and subarachnoid hemorrhage without midline shift. B, E: Preoperative CT scans revealing a 4-mm thick, right fronto-temporal acute subdural hematoma (ASDH) and a left frontal small contusion associated with severe brain swelling and midline shift. C, F: Postoperative CT scans revealing a 10-mm thick, contralateral ASDH with midline shift and mass effect. duced coagulopathy except for a slight increase of fibrinogen. After surgery, the pupils were $5 \mathrm{~mm}$ in diameter respectively, and unresponsive to light. Despite supportive therapy with barbiturate and mild hypothermia, postoperative ICP remained about $21 \mathrm{mmHg}$, and cerebral perfusion pressure was around $68 \mathrm{mmHg}$. He expired 3 days later of brain death.

\section{Discussion}

Decompressive craniectomy is an effective and favorable technique for rapidly reducing ICP in patients with ASDH, ${ }^{20)}$ but decompressive craniectomy may release the tamponade effect and result in brain shift which exposes these patients to the risk of complications caused by developing distant intracranial hemorrhage..$^{4,9,16,18,20)}$ Contralateral epidural and intraparenchymal hematoma have received considerable attention in the literature, ${ }^{1,11,16)}$ but contralateral ASDH is worthy of more attention. This rare complication may lead to potentially life-threatening disorders, if unrecognized. We reviewed the literature to identify cases of contralateral ASDH occurring immediately after evacuation of traumatic $\mathrm{ASDH}$, as summarized in Table $1.1,4,9,18)$ The present case was 1 of 126 cases $(0.8 \%)$ treated at our facility for ASDH.

The pathophysiologic mechanisms of contralateral ASDH occurring after ASDH evacuation are not clear. There is probably no single etiologic factor. Sudden increase in cerebral blood flow (CBF) with defective autoregulation, damage to cerebral vasculature secondary to perioperative parenchymal shift, previously undetected contusion, and bleeding secondary to decompression and coagulopathy have all been proposed as causative factors of delayed intracranial hematomas. ${ }^{6,10,12,17)}$ Reviewing the reported cases, the average age was 52 years, half of the patients were older than 60 years, and two patients were older than 80 years, indicating that the old are more susceptible to contralateral ASDH than the young. In our

Table 1 Characteristics of reported cases of contralateral acute subdural hematoma following traumatic acute subdural hematoma evacuation

\begin{tabular}{|c|c|c|c|c|c|c|c|c|c|c|c|}
\hline \multirow{2}{*}{$\begin{array}{l}\text { Case } \\
\text { No. }\end{array}$} & \multirow{2}{*}{ Author (Year) } & \multirow{2}{*}{$\begin{array}{c}\text { Age } \\
\text { (yrs)/ } \\
\text { Sex }\end{array}$} & \multirow{2}{*}{$\begin{array}{c}\text { Injury } \\
\text { mechanism }\end{array}$} & \multirow{2}{*}{$\begin{array}{l}\text { Preoperative } \\
\text { GCS score }\end{array}$} & \multirow{2}{*}{ Pupils } & \multirow{2}{*}{$\begin{array}{l}\text { Brain } \\
\text { shift* } \\
(\mathrm{mm})\end{array}$} & \multirow{2}{*}{$\begin{array}{l}\text { Brain } \\
\text { swelling }\end{array}$} & \multirow{2}{*}{ Coagulopathy } & \multicolumn{2}{|c|}{$\begin{array}{c}\text { Time to } \\
\text { surgery (hrs) }\end{array}$} & \multirow{2}{*}{ GOS } \\
\hline & & & & & & & & & First & Second & \\
\hline 1 & $\begin{array}{l}\text { Ban et al. } \\
(1991)^{1)}\end{array}$ & $62 / \mathrm{F}$ & traffic & 7 & bi-mydriasis & ND & yes & no & 3 & IM & $\mathrm{D}$ \\
\hline 2 & & $72 / \mathrm{M}$ & traffic & 5 & bi-mydriasis & ND & yes & no & 0.5 & IM & $\mathrm{D}$ \\
\hline 3 & $\begin{array}{l}\text { Matsuno et al. } \\
(2003)^{9)}\end{array}$ & $31 / \mathrm{M}$ & fall & 6 & bi-mydriasis & 24 & yes & no & 1 & IM & SD \\
\hline 4 & & $40 / \mathrm{M}$ & traffic & 3 & bi-mydriasis & 10.3 & yes & no & 3 & IM & SD \\
\hline 5 & & $19 / \mathrm{M}$ & fall & 4 & uni-mydriasis & 29 & yes & no & 5 & IM & VS \\
\hline 6 & $\begin{array}{c}\text { Tomycz et al. } \\
(2010)^{18)}\end{array}$ & $81 / \mathrm{F}$ & fall & 15 & normal & 23 & no & yes & ND & IM & GR \\
\hline 7 & $\begin{array}{l}\text { Fridley et al. } \\
(2011)^{4)}\end{array}$ & $85 / F$ & fall & 15 & normal & 16 & no & no & ND & IM & GR \\
\hline 8 & Present case & $26 / \mathrm{M}$ & traffic & 8 & bi-mydriasis & 21 & yes & no & 3 & IM & $\mathrm{D}$ \\
\hline
\end{tabular}

${ }^{*}$ Together with bilateral brain shift. bi-mydriasis: bilateral mydriasis, D: dead, F: female, GCS: Glasgow Coma Scale, GOS: Glasgow Outcome Scale, GR: good recovery, IM: immediate, M: male, ND: not described, SD: severe disability, uni-mydriasis: unilateral mydriasis, VS: vegetative state. 
opinion, this may be explained by the fact that the brain parenchyma severely compressed by large hematoma had lost structural elasticity, that is, significant re-expansion of brain did not occur immediately after operation due to increasing brain atrophy and subdural space with age, caused mechanical parenchymal shift and subdural space detachment from the cerebral parenchyma opposite to the craniectomy, so the contralateral bridging veins consequently ruptured and bled resulting in ASDH formation. An 81-year-old woman with coagulopathy of INR of 4.2 developed a contralateral ASDH with midline shift shortly after craniotomy for evacuation of a traumatic right ASDH. ${ }^{18)}$ In addition, older patients have dura that is more adherent to the skull inner table, ${ }^{4,13)}$ which increases the effects of vacuum pressure and may predispose older patients to tear bridging veins after hematoma evacuation. In our case, bleeding from a cortex artery in the frontal lobe contusion was demonstrated during the second operation, which caused hemorrhage into the contralateral contusion secondary to sudden increase in CBF associated with defective autoregulation or tamponade effect removal, and contributed to the formation of ASDH. These are the most likely causative factors in young patients.

Intraoperative brain swelling was found after evacuation of traumatic ASDH in 6 of the 8 reported cases (75\%) with poor prognosis. Intraoperative brain swelling is a warning sign of contralateral ASDH formation in traumatic patients, ${ }^{16)}$ so neurosurgeons should try to detect the development of contralateral hematoma and prevent clinical deterioration, particularly in the absence of clinical evidence of brain swelling. Postoperative routine CT is a reliable way to evaluate this potential complication for a comprehensive strategy of prompt treatment.4,16) This need is demonstrated clearly by two cases with the development of contralateral hematoma without intraoperative brain swelling. Any unexplained increase in ICP warrants immediate CT, which is the best choice to establish the diagnosis for intervention for this evolving pathology. Contralateral hematomas can be easily and precisely identified using intraoperative sonography, which could lead to early amelioration of the life-threatening mass effect caused by contralateral expansion, ${ }^{1,7}$ but comparison with CT findings is necessary to prospectively evaluate the efficiency of intraoperative sonography.

How can we prevent this devastating complication? We suggest that early and gradual decompression could prevent the sudden brain shift and decreased tamponade effect. Decompression with burr-hole craniotomy rapidly followed by decompressive craniectomy may help to decrease the risk of contralateral hemorrhage in our experience, but there are several other favorable measures as follows. Dural netted incision to prevent severe brain swelling involves incision of the dura in a netted shape to evacuate hematoma and reduce brain shift. ${ }^{5)}$ Dural incision step by step to remove the hematoma so as to minimize the changes in pressure. Correct head position to aggravate brain shift, especially in the presence of no significant re-expansion of brain tissue. ${ }^{12}$

In conclusion, contralateral ASDH following evacua- tion of traumatic ASDH is a rare but potentially lethal complication, so neurosurgeons should try to detect such contralateral hematoma formation and prevent clinical deterioration. Various measures may prevent this devastating complication. More experience is needed for further understanding of the pathophysiology and treatment.

\section{Conflicts of Interest Disclosure}

The authors have no personal financial or institutional interest in any of the drugs, materials, or devices in the article.

\section{References}

1) Ban M, Agawa M, Fukami T: Delayed evolution of post-traumatic contralateral extracerebral hematoma after evacuation of initial hematoma. Neurol Med Chir (Tokyo) 31: 927-930, 1991

2) Brisman MH, Bederson JB, Sen CN, Germano IM, Moore F, Post KD: Intracerebral hemorrhage occurring remote from the craniotomy site. Neurosurgery 39: 1114-1122, 1996

3) Danish SF, Barone D, Lega BC, Stein SC: Quality of life after hemicraniectomy for traumatic brain injury in adults. A review of the literature. Neurosurg Focus 26(6): E2, 2009

4) Fridley J, Thomas J, Kitagawa R, Chern J, Omeis I: Immediate development of a contralateral acute subdural hematoma following acute subdural hematoma evacuation. J Clin Neurosci 18: 422-423, 2011

5) Guilburd JN, Sviri GE: Role of dural fenestrations in acute subdural hematoma. J Neurosurg 95: 263-267, 2001

6) Harada K, Ohtsuru K, Nakayama K, Takagi S, Shigemori M, Tokunaga T, Sugita Y, Torigoe R: Contralateral development of acute subdural hematoma following surgery for chronic subdural hematoma-case report. Neurol Med Chir (Tokyo) 32: 969-971, 1992

7) Huang AP, Chen YC, Hu CK, Lin TK, Huang SJ, Tu YK, Tsai YH: Intraoperative sonography for detection of contralateral acute epidural or subdural hematoma after decompressive surgery. J Trauma 70: 1578-1579, 2011

8) Kontopoulos V, Foroglou N, Patsalas J, Magras J, Foroglou G, Yiannakou-Pephtoulidou M, Sofianos E, Anastassiou H, Tsaoussi G: Decompressive craniectomy for the management of patients with refractory hypertension: should it be reconsidered? Acta Neurochir (Wien) 144: 791-796, 2002

9) Matsuno A, Katayama H, Wada H, Morikawa K, Tanaka K, Tanaka H, Murakami M, Fuke N, Nagashima T: Significance of consecutive bilateral surgeries for patients with acute subdural hematoma who develop contralateral acute epi- or subdural hematoma. Surg Neurol 60: 23-30, 2003

10) Modesti LM, Hodge CJ, Barnwell ML: Intracerebral hematoma after evacuation of chronic extracerebral fluid collections. Neurosurgery 10(6 Pt 1): 689-693, 1982

11) Mohindra S, Mukherjee KK, Gupta R, Chhabra R, Gupta SK, Khosla VK: Decompressive surgery for acute subdural haematoma leading to contralateral extradural haematoma: a report of two cases and review of literature. Br J Neurosurg 19: 490-494, 2005

12) Moon KS, Lee JK, Kim TS, Jung S, Kim JH, Kim SH, Kang SS: Contralateral acute subdural hematoma occurring after removal of calcified chronic subdural hematoma. J Clin Neurosci 14: 283-286, 2007

13) Murzin VE, Goriunov VN: [Study of the strength of the adherence of the dura mater to the bones of the skull]. Zh Vopr 
Neirokhir Im N N Burdenko (4): 43-47, 1979 (Russian)

14) Seelig JM, Becker DP, Miller JD, Greenberg RP, Ward JD, Choi SC: Traumatic acute subdural hematoma: major mortality reduction in comatose patients treated within four hours. N Engl J Med 304: 1511-1518, 1981

15) Stone JL, Lowe RJ, Jonasson O, Baker RJ, Barrett J, Oldershaw JB, Crowell RM, Stein RJ: Acute subdural hematoma: direct admission to a trauma center yields improved results. J Trauma 26: 445-450, 1986

16) Su TM, Lee TH, Chen WF, Lee TC, Cheng CH. Contralateral acute epidural hematoma after decompressive surgery of acute subdural hematoma: clinical features and outcome. $J$ Trauma 65: 1298-1302, 2008

17) Su TM, Shih TY, Yen HL, Tsai YD: Contralateral acute subdural hematoma occurring after evacuation of subdural hygroma: case report. J Trauma 50: 557-559, 2001
18) Tomycz ND, Germanwala AV, Walter KA: Contralateral acute subdural hematoma after surgical evacuation of acute subdural hematoma. J Trauma 68: E11-12, 2010

19) Wilberger JE, Harris M, Diamond DL: Acute subdural hematoma: morbidity, mortality, and operative timing. J Neurosurg 74: 212-218, 1991

20) Yang XF, Wen L, Shen F, Li G, Lou R, Liu WG, Zhan RY: Surgical complications secondary to decompressive craniectomy in patients with a head injury: a series of 108 consecutive cases. Acta Neurochir (Wien) 150: 1241-1248, 2008

Address reprint requests to: Renya Zhan, MD, the Department of Neurosurgery, First Affiliated Hospital, College of Medicine, Zhejiang University, No. 79 Qingchun Road, Hangzhou City, 310003 Zhejiang Province, PRC. 